\title{
PLACING THE GUIDE STAR CATALOG ON THE HIPPARCOS SYSTEM
}

\author{
U. BASTIAN ${ }^{1}$, B. BUCCIARELLI ${ }^{2,3}$, J. HAYES ${ }^{2}$, B. M. LASKER ${ }^{2}$, J. E. MORRISON ${ }^{2}$, S.
} RÖSER ${ }^{1}$, R. L. SMART ${ }^{2}$, C. R. STURCH ${ }^{4}$ AND L. G. TAFF ${ }^{2}$

${ }^{1}$ Astronomisches Rechen-Institut, Mönchhofstr. 12-14, 69120 Heidelberg, Germany

${ }^{2}$ STScI, 3700 San Martin Drive, Baltimore, MD 21218 USA

${ }^{3}$ On leave from Torino Observatory, Torino, Italy

${ }^{4}$ Computer Sciences Corp., Astronomy Programs, STScI, Baltimore, MD 21218 USA

The HIPPARCOS Catalogue, linked to the system of extra-galactic radio sources, will soon be the reference system in optical astronomy. The Guide Star Catalog (GSC; Lasker et al. AJ 99, 2019ff, 1990), with its 18 million objects, already serves most astronomers for the planning of small field observations, optical identifications, and the reduction of CCD images. The utility of the GSC will be greatly enhanced if it is transformed to the HIPPARCOS system and its remaining systematic errors minimized. To do so we will use the forthcoming TYCHO Reference Catalogue (Röser \& Høg, in Workshop on Databases for Galactic Structure, eds. Davis Philip, Hauck \& Upgren, pp. 137, 1993). The reductions will use a combination of the mask and sub-plate methods (Taff, AJ 98, 1912, 1989; Taff, Lattanzi \& Bucciarelli ApJ 361, 667, 1990), the co-location method (Bucciarelli, Lattanzi \& Taff, ApJ Suppl. 84, 91, 1993), the new "fixed number of stars filter" of Röser, Bastian \& Kuzmin (in press in the proceedings of IAU 148, 1994), and the infinitely overlapping circles (IOC) version of the latter (Taff, Bucciarelli \& Lattanzi, ApJ 358, 359, 1990; Bucciarelli, Taff \& Lattanzi, J. Stat. Comp. Sim. 48, 29, 1993). We present in the table below test results using the PPM as the reference catalog (typically only 4 sub-plates per plate; 25 stars for the Square Filter; 16 stars per circle for the IOC method). The first line presents the estimates for $\sigma_{\alpha} \cos \delta$ and $\sigma_{\delta}$, both in arcsec, relative the to PPM Suppl (Röser, Bastian \& Kuzmin, A\&A Suppl. 105, 301, 1994); the second line contains them based on plate overlaps (all for the $-30^{\circ}$ declination zone). When comparing the numbers note that in the first line the GSC and PPM Suppl (0."12) errors are contributing whereas in the second line the GSC errors are present twice (in the rms sense).

\begin{tabular}{||l|c|c|c|c|c||}
\hline & $\begin{array}{l}\text { GSC } \\
1.0\end{array}$ & MASK & $\begin{array}{c}\text { SUB- } \\
\text { PLATE }\end{array}$ & $\begin{array}{l}\text { SQUARE } \\
\text { FILTER }\end{array}$ & IOC \\
\hline PPMSuppl & $0.72,0.60$ & $0.34,0.35$ & $0.57,0.56$ & $0.35,0.34$ & $0.33,0.30$ \\
\hline Overlaps & $0.87,0.62$ & $0.80,0.60$ & $1.10,0.49$ & $0.64,0.44$ & $0.57,0.31$ \\
\hline
\end{tabular}

Further work is required to select the optimum method (which may vary with the plate). However, it is clear that any modern small-area reduction procedure, based on a high quality, dense reference catalog, produces results far superior to global Schmidt plate modeling. Research supported by NASA Contract NAS5-32496 and the STScI Collaborative Visitors Program. 